\title{
Access to health care for gaming disorder: A patient-centred approach to address the continuum of need
}

Jennifer J Park

University of Auckland

Laura Wilkinson-Meyers

University of Auckland

Daniel L King

Flinders University

Simone Rodda ( $\nabla$ s.rodda@auckland.ac.nz )

University of Auckland https://orcid.org/0000-0002-7973-1003

Research article

Keywords: gaming disorder, treatment, internet gaming, screening, intervention

Posted Date: October 27th, 2020

DOl: https://doi.org/10.21203/rs.3.rs-30827/v2

License: (a) (i) This work is licensed under a Creative Commons Attribution 4.0 International License.

Read Full License 


\section{Abstract}

Background Problematic gaming, including its most serious form known as gaming disorder (GD) in the ICD-11, affects approximately $1-3 \%$ of the population and can have significant negative impacts on health and wellbeing. Research to determine the optimal health system responses to GD remains limited. This study aimed to identify and describe the key components of a patient-centred approach to prevention, early intervention, and treatment of GD.

Methods Online interviews were conducted with 20 adults ( $90 \%$ male; $\mathrm{M}_{\mathrm{age}}=23 \mathrm{y}$ ) currently seeking help for GD. The interview protocol was guided by a health care access framework which investigated participants' experiences and needs related to accessing professional support. Transcripts were analysed in NVivo using qualitative content analysis to systematically classify participant data into the themes informed by this framework.

Results Although the characteristics and resultant harms of GD were broadly recognised, participants indicated that these issues may not always be viewed by healthcare providers as a serious problem that requires intervention or that could be addressed through treatment. This highlights a significant barrier to access at the beginning of the patient journey when patients may perceive that they have a legitimate need for care and seek a relevant, tailored, and safe service to meet this need. Participants described the essential components of an effective health service for GD as including: valid and reliable screening tools for GD; practitioners with specialist knowledge of GD that includes, or is strongly informed by, lived experience of peers; and access to a multimodal system of intervention, including self-help, internet and face-to-face options that allow gamers to transition between types and intensity of support.

Conclusion A comprehensive health care approach for gaming disorder is currently in its infancy and there are numerous service access and delivery issues still to be resolved. This study highlights the importance of involving individuals with GD in developing solutions that are fit for purpose and address the spectrum of intervention needs. These findings recommend a stepped GD healthcare system that adheres to evidence-based practice tailored to each individual and the implementation of standard assessment and routine outcome monitoring.

\section{Background}

Gaming disorder is recognised as a mental health issue that impacts approximately $1-3 \%$ of people internationally (1-4). The condition is associated with physical (e.g., sleep disruption), psychological (e.g., anxiety and depression), social (e.g., impaired quality of relationships) and legacy harms such as reduced employment or educational attainment (5-7). Gaming disorder is highly comorbid with anxiety, depression, ADHD, social phobia, and anxiety, and appears to be more prevalent in male gamers $(8,9)$. The concept of problem gaming as an addictive disorder has been the topic of extensive debate $(2,10$ 18). Following a provisional status for 'internet gaming disorder' in the DSM-5 (American Psychiatric Association, 2013), gaming disorder was officially adopted at the World Health Assembly in May 2019 as 
a diagnosis in the eleventh edition of the International Classification of Diseases (ICD-11) (19). Gaming disorder within these health classification systems is characterized by persistent gaming behaviour, impaired control over gaming, and functional impairment due to gaming for a period of at least 12 months in most instances.

Recent systematic reviews and meta-analyses have examined the evidence quality and effectiveness of GD prevention (20-23) and treatment (24-27). To date, research on prevention has tended to focus on school-based prevention programs. Much of the work in this area has been conducted in Asian settings $(28,29)$, including South Korea and China, where there have been parallel developments in trialling targeted technology-restriction measures including content filters and gaming time limits aimed particularly at younger users (30-33). In terms of treatment, the most common approach has been cognitive-behavioural therapy (CBT), usually delivered in brief individual and group-based formats, and other non-CBT psychotherapeutic interventions $(24-26,34,35)$. CBT for GD may be an effective short-term intervention for reducing GD and depressive symptoms, but more studies with follow-up are needed to assess longer-term gains $(34,36)$. Pharmacological interventions have predominantly employed antidepressants (i.e., bupropion and escitalopram), but their effectiveness is currently unclear due to lack of controlled trials (14). Some treatment centres provide brief voluntary retreats, which typically involve abstinence (so-called 'detox') from digital technologies, group therapy and social activities, but these options may be financially burdensome and have limited evidence for their long-term efficacy.

The growing body of literature examining early intervention and treatment for GD highlights several important gaps $(24,25,29,35)$. Zajac et al.'s $(25)$ review of 15 studies reported that the majority had targeted school age samples with just six studies involving adults. The majority of participants were male and almost all were conducted in university settings, however there was some heterogeneity in terms of problem severity (mild, moderate and severe problems) and time spent gaming (ranging daily to weekly sessions, and very low to high levels of gaming time). Treatment was often brief intensive, being conducted over 4 to 8 weeks, and almost all were delivered via face-to-face consultation. Other reviews $(24,35)$ have reported most studies have been conducted in East Asian settings, indicating a need for cross-cultural perspectives. There are well-documented problems of validity across a wide range of GD tools, including those employed in intervention studies $(37,38)$. A related problem is that many intervention studies, particularly those in Asia, have relied on problematic internet use measures to evaluate gaming. These measurement inconsistencies add to problems identified with specific criteria listed in the IGD classification, particularly the relatively low specificity of the tolerance and mood modification criteria (39). Together, these reviews suggest that GD interventions may be enhanced by a range of improvements and wider consideration of alternative treatment approaches and delivery methods.

Although many individuals and their families experience problem gaming-related harm, very few will seek professional help $(23,40)$. A longitudinal study involving over 4000 adults in Canada reported just 3 percent of the 201 with gaming problems had sought professional help over a 5-year period (41). The type of help surveyed included a family physician, psychologist, psychiatrist, counseling service or 
telephone helpline. Rates of professional treatment-seeking for other addictive behaviours is approximately $10 \%$ (42), much higher than is reported for GD. The reason for the low rate of treatment seeking may be due to structural issues such as the homogeneity of available treatments (intensive and face-to-face). In addition, individual factors related to GD may delay or inhibit treatment seeking, such as low insight, procrastination, impulsivity, and shyness or introversion (43-46). Another possibility is that some individuals with gaming-related problems may initially seek out information, social support and assistance from less formal, convenient sources, such as online support groups (47).

Another important consideration for interventions is the complex nature of GD cases. Given high rates of co-morbidity and GD (e.g., depression, anxiety, substance use and gambling disorder) it may be that people with GD attend other services for more acute issues and therefore do not report seeking help for GD (46). Lau et al. (48) for example, examined the records of 5820 clinically referred youth in the Canadian mental health system and reported that moderate to severe problematic gaming was reported by $13 \%$ of the sample; however, most of the sample had been referred for issues including threat or danger to self or others, or other psychiatric symptoms. GD treatment is also challenged by the ubiquity of gaming opportunities and the common perception among patients that maintaining their commitment to the gaming world is preferable to life outside of that context (49). This suggests a need to examine how health care can be provided so that appropriate supports are readily available to anyone concerned about their gaming and is designed appropriately to reflect a range of client insights, preferences, and goals.

Taken together, the evidence suggests a need to broaden the scope of research into GD interventions to address the complexities and varied needs of individuals. To address complex issues related to GD health care, the current study undertook interviews with people experiencing problems with gaming to gather their views of the components of an effective integrated health care approach. Although GD prevalence in New Zealand (NZ) is currently unclear, there is evidence of problematic gamers in this region (50) and surveys of psychiatrists and mental health professionals that suggest NZ gamers and their support networks may seek help via addiction and related services $(51,52)$. The aims of this study were to: (i) describe the experiences and needs of people seeking help for GD (inclusive of gaming problems); and (ii) identify the optimal components of a health care system to support prevention, early intervention and treatment of GD.

\section{Methods}

\section{Participants}

Participants were drawn from a larger study examining the impact of a brief intervention for GD (53). The larger study involved 50 gamers who reported an intention to limit or reduce their gaming in the next 30 days. Participants were also required to be aged 18 years or older and not currently seeking treatment. The current study involved sequential recruitment of the first 20 participants willing to engage in a semistructured online interview examining the needs, experiences, and preferences for treatment-seeking in 
New Zealand. Recruitment occurred over a one-month period between April and May 2019 through social media advertising, posters in the Auckland community (university, schools, supermarkets) and word of mouth. While there was no specific remuneration for the current study, all participants received a NZ\$50 shopping voucher for completing follow-up evaluation as part of the main study. The research was approved by the University of Auckalnd Human Participants Ethics Committee (022614).

Table 1 presents a summary of participant information. The majority of participants were male $(n=18$, $90 \%$ ). The average age of participants was 23 years (range: 20 to 35 years). The sample primarily identified as New Zealand European $(n=10,50 \%)$ and as Asian $(n=7,35 \%)$. Using the Gaming Addiction Scale-21 (54) to screen for problematic gaming, 16 participants were classified as problematic gamers (endorsed four or more of seven items) which included 5 participants classified as having GD (endorsed all seven items). Four participants indicated concerns about their gaming but did not meet the GAS cutoff score. The average hours spent online per week was $29(S D=23)$ with an average frequency of 9 sessions per week $(S D=4.7)$.

Table 1: Participant characteristics at baseline 


\begin{tabular}{|c|c|c|c|c|c|c|c|}
\hline ID & $\begin{array}{c}\text { Age \& } \\
\text { Gender }\end{array}$ & Gender & Employment & $\begin{array}{l}\text { GAS } \\
\text { criteria }\end{array}$ & $\begin{array}{l}\text { Frequency } \\
\text { gaming } \\
\text { p/w }\end{array}$ & $\begin{array}{c}\text { Hours } \\
\text { gaming } \\
\mathrm{p} / \mathrm{w}\end{array}$ & $\begin{array}{l}\text { Psychological } \\
\text { Distress (K6) }\end{array}$ \\
\hline 1 & 20 & Female & $\begin{array}{l}\text { Student (not } \\
\text { employed) }\end{array}$ & Unmet & 17 & 6 & 24 \\
\hline 2 & 20 & Male & $\begin{array}{l}\text { Unemployed, not } \\
\text { looking for work }\end{array}$ & $\begin{array}{l}\text { Problem } \\
\text { gaming }\end{array}$ & 11 & 21 & 12 \\
\hline 3 & 25 & Male & Employed part time & $\begin{array}{l}\text { Problem } \\
\text { gaming }\end{array}$ & 18 & 70 & 21 \\
\hline 4 & 24 & Male & $\begin{array}{l}\text { Student (not } \\
\text { employed) }\end{array}$ & $\begin{array}{l}\text { Problem } \\
\text { gaming }\end{array}$ & 9 & 74 & 22 \\
\hline 5 & 21 & Male & $\begin{array}{l}\text { Student (not } \\
\text { employed) }\end{array}$ & GD & 11 & 40 & 15 \\
\hline 6 & 36 & Male & Other (not stated) & GD & 7 & 74 & 7 \\
\hline 7 & 21 & Male & $\begin{array}{l}\text { Student (not } \\
\text { employed) }\end{array}$ & $\begin{array}{l}\text { Problem } \\
\text { gaming }\end{array}$ & 5 & 10 & 19 \\
\hline 8 & 21 & Male & Employed part time & GD & 7 & 47 & 19 \\
\hline 9 & 20 & Male & $\begin{array}{l}\text { Unemployed, } \\
\text { looking for part } \\
\text { time work }\end{array}$ & $\begin{array}{l}\text { Problem } \\
\text { gaming }\end{array}$ & 10 & 34 & 18 \\
\hline 10 & 23 & Male & Employed part time & GD & 10 & 18 & 18 \\
\hline 11 & 22 & Male & Employed full time & $\begin{array}{l}\text { Problem } \\
\text { gaming }\end{array}$ & 18 & 28 & 21 \\
\hline 12 & 21 & Male & $\begin{array}{l}\text { Student (not } \\
\text { employed) }\end{array}$ & $\begin{array}{l}\text { Problem } \\
\text { gaming }\end{array}$ & 4 & 4 & 17 \\
\hline 13 & 22 & Male & $\begin{array}{l}\text { Unemployed, not } \\
\text { looking for work }\end{array}$ & $\begin{array}{l}\text { Problem } \\
\text { gaming }\end{array}$ & 9 & 17 & 14 \\
\hline 14 & 21 & Female & Employed part time & Unmet & 3 & 5 & 10 \\
\hline 15 & 22 & Male & Employed part time & Unmet & 7 & 14 & 13 \\
\hline 16 & 26 & Male & $\begin{array}{l}\text { Student (not } \\
\text { employed) }\end{array}$ & $\begin{array}{l}\text { Problem } \\
\text { gaming }\end{array}$ & 7 & 13 & 21 \\
\hline 17 & 20 & Male & $\begin{array}{l}\text { Unemployed, } \\
\text { looking for part } \\
\text { time work }\end{array}$ & GD & 16 & 58 & 14 \\
\hline 18 & 21 & Male & Employed full time & $\begin{array}{l}\text { Problem } \\
\text { gaming }\end{array}$ & 11 & 25 & 17 \\
\hline 19 & 20 & Male & Employed part time & Unmet & 2 & 10 & 11 \\
\hline 20 & 32 & Male & Employed full time & $\begin{array}{l}\text { Problem } \\
\text { gaming }\end{array}$ & 7 & 10 & 23 \\
\hline
\end{tabular}

GAS: Game Addiction Scale; K6: Kessler-6.

\section{Procedure}

All participants completed a participant information sheet and informed consent form in Qualtrics survey software. This included completion of demographic (age, gender, employment, ethnicity) and gamingrelated measures. The survey included the 21 -item Game Addiction Scale (54) which measures seven dimensions of problem gaming (i.e., salience, tolerance, mood modification, relapse, withdrawal, conflict and problems). Participants also completed the Time Line Follow Back $(55,56)$ to determine the duration and frequency of gaming, and the Kessler 6 measure of non-specific psychological distress (57) 
A semi-structured interview schedule was developed based on the components of the patient-centred model for accessing health care by Levesque (2013) and colleagues (58). As indicated in Figure 1, this access framework highlights the important relationship between the design of health services and the capabilities of those who seek help to facilitate access to appropriate health care and improved health.

The purpose of the interviews was to identify support and treatment needs and preferences specific to gaming disorder. The first part of the interview schedule focused on the capabilities of gamers, or the individual barriers and facilitators to help seeking. This included the questions "I understand that you are interested in doing something about your gaming. What made you make this decision?" and "What might stop you from getting help? What could help address these barriers?" Additional prompts were included in the semi-structured interview schedule to continue the conversation if it became stalled (i.e., the participant needed more context on the nature of the questions). These included: reasons for wanting to change gaming and reasons for seeking help; barriers and facilitators to help seeking and knowledge of services. The second part of the interview schedule focused on support and service needs. Specifically, participants were asked: "Consider what services that could be developed for people wanting help for gaming. Let's say you decide you want to seek help in the future. What would be important to you in a service?" Prompts included accessibility (cost, waiting times, travel, location, type of support), quality (treatment types, qualifications, relationships, desired resources) and equity (considerations for culture, age, gender and social disadvantage).

Interviews were conducted by a postgraduate population health student under supervision. The interviewer was trained in motivational interviewing and the administration of a semi-structured interview schedule. Interviews were by appointment and delivered via the participants' preferred instant messaging platform (Facebook Messenger, Skype, WhatsApp). Instant messaging was used instead of recording video call to provide greater participant anonymity, increase personal disclosure, and provide more coherent verbal expression for qualitative analysis. Transcripts were created by copying and pasting the online conversation into a word document. The average time per interview was 90 minutes (range 40 to 180 minutes) and the average word count was 839 (range 377 to 1988).

\section{Data analysis and application of framework}

We applied the conceptual framework of patient-centred access to health care to understand how services can meet the needs of people with GD (58). This framework systematically describes the client and provider factors necessary to facilitate the entire patient experience from the point of identifying a health care need to the ultimate achievement of improved health outcomes. (see Figure 1). From the client perspective, the framework outlines five processes that influence access to support and treatment. These include the ability to perceive a need for treatment (including health literacy, health beliefs and knowledge of services); ability to seek, reach and pay for treatment as well as an ability to engage (facilitating minimal attrition and maximum adherence). From the treatment provider perspective, client access to health care is influenced by their approachability (service information, screening tools), acceptability (values and culture of the organisation and support for client autonomy), availability 
(physical location, mode of delivery, qualifications of provider and flexible opening hours), affordability (time and resource costs) and appropriateness (delivery of evidence-based treatment tailored to client need). A qualitative content analysis (59) was used to group our data into this framework. This method was selected because we wanted to systematically classify participant data into themes guided by an established framework (59).

NVivo software was used to assist the qualitative content analysis. Transcripts were read twice for familiarity with the data and initial codes developed. Two complete transcripts were coded by two researchers (JP and SR) and the codes discussed and any disagreements resolved. Common codes were grouped by the first author into themes that were adjusted to accommodate additional data. As outlined by Hsieh and Shannon (59), this data was then grouped into broad categories which reflected the selected framework. Data that could not be coded into one of the broad categories were re-examined to describe different manifestations of treatment needs and these were merged into the framework and noted accordingly. The findings was structured according to: (1) participant ability to percieve, seek, reach, pay and engage with health care which is supported with illustrative quotes, and (2) the proposed components of a health care system response specific to GD. We reported the proportion of participant statements ( $n=568$ statements that were coded) that align with each of the five components of the framework. All 20 participants provided at least one statement that was relevant to the framework. Where quotes were used, these were de-identified to ensure participant anonymity. Quotes were also cleaned to improve readability (i.e., spelling and punctuation) as well as clarity (i.e., grammar corrected).

\section{Results}

\section{Treatment needs and preferences}

Table 2 presents a summary of the five components of the framework from the client perspective. The most frequently discussed participant experiences related to ability to seek help with relatively less discussion on ability to pay for services. The ability to perceive a need for support was focused on health beliefs and health literacy and ( $10 \%$ of statements from the 20 participants). Health beliefs involved a cycle of minimising potential risk associated with uncontrolled gaming as well as the perceived seriousness of problem gaming-related harm. As indicated in Table 1, participants stated that excessive gaming caused a range of harms from reduced functioning across health, work, sleep and social life. While there were many different reported reasons for change, this was at times coupled with the belief that the problem was not as serious as other addictions despite very large amounts of time being spent on gaming. For some participants, there were co-occurring issues such as alcohol or cannabis or mental health issues that were perceived as more acute and/or deserving of attention. Even though they were seeking help, these participants reported a general perspective that GD was not real, trivial, or a "soft" addiction. Participants indicated they had a reasonable understanding of the characteristics of GD including its nature, prevalence, and resultant harms.

Table 2: Mapping participant experiences against the five constructs 
Page 9/24 


\begin{tabular}{|c|c|c|}
\hline Construct & Participant experiences & Indicative quotes \\
\hline 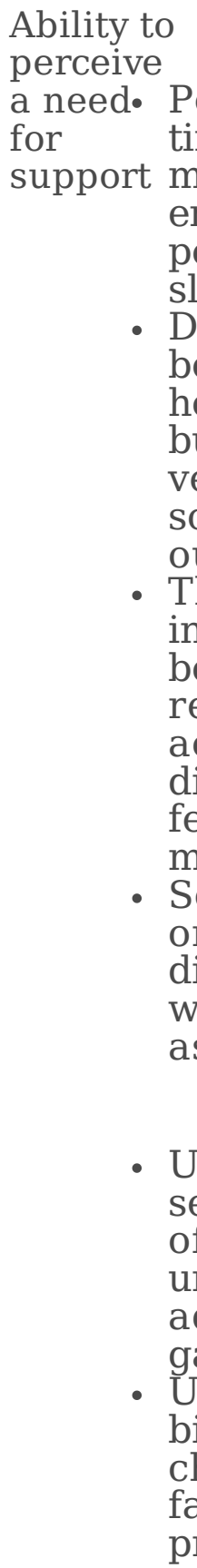 & $\begin{array}{lc}\text { Health beliefs } \\
\text { •rceived harm included wasted } \\
\text { me, energy, ability to focus on } \\
\text { pre meaningful achievement, } \\
\text { trenched lifestyle, ignoring } \\
\text { grsonal hygiene or disrupted } \\
\text { eep. }\end{array}$ & 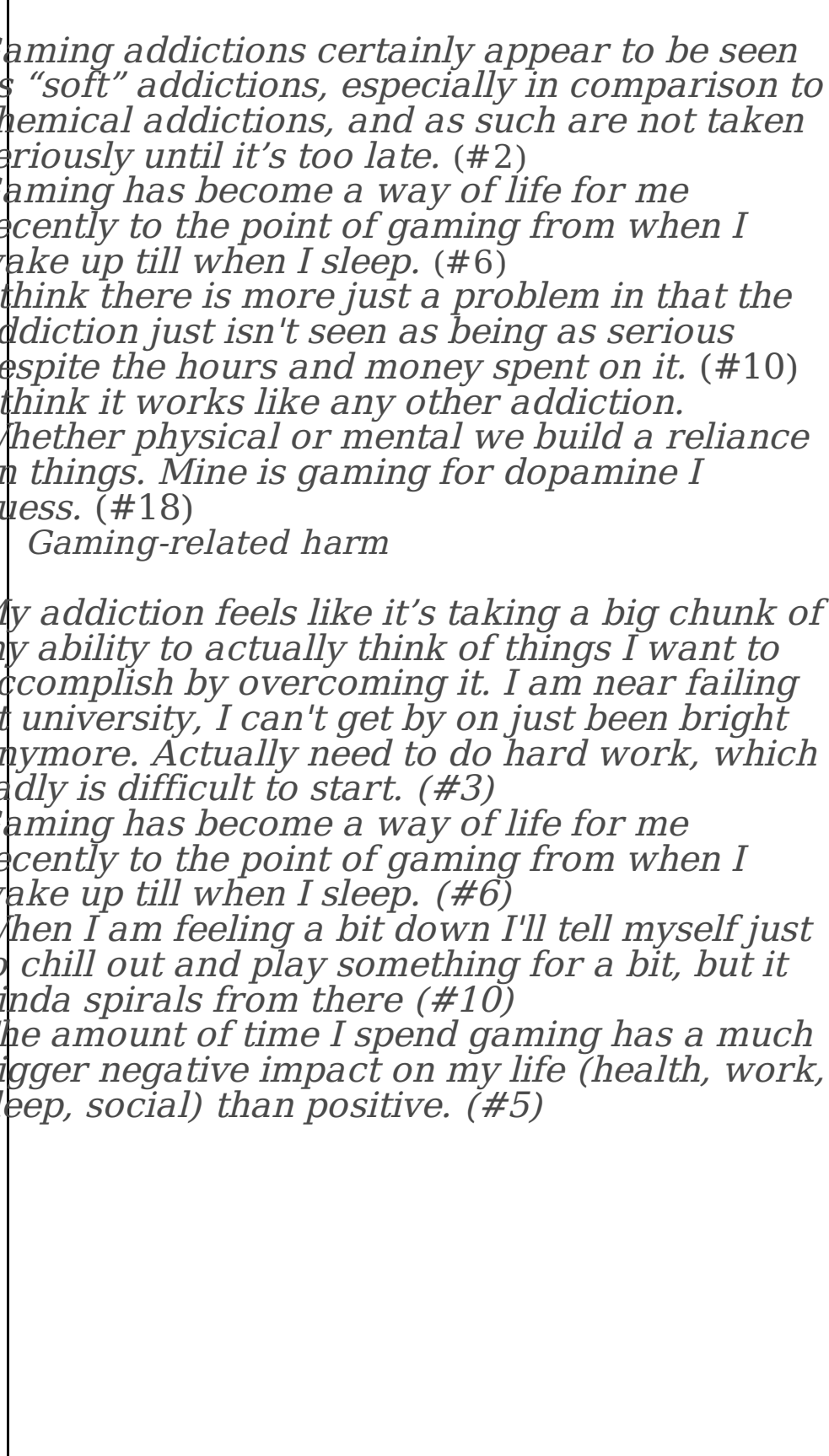 \\
\hline $\begin{array}{l}\text { Ability to } \\
\text { seek } \\
\text { support L } \\
\\
\\
\text { Se } \\
\text { h }\end{array}$ & $\begin{array}{l}\text { Knowledge of services } \\
\text { mited knowledge of where to } \\
\text { ek help. Limited knowledge of } \\
\text { कw treatment works. } \\
\text { Appropriateness of help seeking } \\
\text { perception that services alreadtya } \\
\text { pe too much need and that } \\
\text { aming is not as important or } \\
\text { armful as other issues. } \\
\text { \$sociated view that services are } \\
\text { qable or unwilling to make time } \\
\text { r gaming issues. } \\
\text { фcating gaming treatment with } \\
\text { qrceived more serious issues }\end{array}$ & $\begin{array}{l}\text { don't know where to seek help for a potential } \\
\text { qming addiction. I don't know of any a vailable } \\
\text { rrvices for specifically this. (\#2) } \\
\text { think if it was made known that these other } \\
\text { tograms could also assist with things such as } \\
\text { gaming habit it would help people like me } \\
\text { ke the issue more seriously because until } \\
\text { cently it never seemed a serious enough issue } \\
\text { gt to the other problems these programs deal } \\
\text { th. (\#7) } \\
\text { eople I know have told me that I should "solve } \\
\text { problem" and that "I need to stop gaming" } \\
\text { couraged me to say seek a service to assist in } \\
\text { e matter. (\# 5) }\end{array}$ \\
\hline
\end{tabular}

Page 10/24 
discourages help-seeking.

- Services do not convey a knowledge of gaming culture or specific approaches to treatment. Other programs dedicated to helping people

- Fuiends, partners, and family oyercome personal issues tend to be focused on provided encouragement to gamemore serious problems such as mental health, less, but limited encouragement, living conditions, or family issues, and therefore support or motivation to seek helpthlese programs don't advertise the fact that or engage in behaviour change. they could help with something like this. (\#7)

\section{Stigma and shame}

- A feeling of shame or erhbarrassment that help is needed and that the issue is bÆyond self-management.

- Help seeking is not typical annongst gamers and the gaming culture does not support leaving gaming networks.

\section{Age and gender appropriate} services

- Pфrception that treatment may no be age or gender appropriate.

This includes the way informationa is currently provided (needs to beand didn't have an understanding of the factors brief and targeted) as well as the that relate to addiction enough to actually help type of information conveyed

(e.g., relevant to young adults).

- Recognise that females have gaming problems too and likely experience additional stigma.

\section{Desire for autonomy}

- A desire to choose personal gaming reduction goals (stay the same, reduce, abstain) and to determine the speed of change.

- A preference to avoid treatment that does not respect individual chloice. Interest in selfmanagement and a do it yourself approach.

- Preference to be treated as an adult and able to make own decisions.

Ability to reach for support F\&w service options means travelling distances to receive help. Ideally it is available within own town or region.

- Willing to travel around 30 minutes to receive treatment. Must have access with public transport

- Willing to wait for an appointmen because it is not generally acute or life threatening. Acceptable dictive behaviour, only physical addiction,

- Yeah of course there's stigma around it. It's quite embarrassing, especially today's university students since my friends play the sa me amount as I do it isn't embarrassing within our circle however outside of that people will always question and shame it. (\#8)

- I feel like people would perceive me in a strange/snowflake way if I were to get help for 'playing too much games'. (\#5)

A pyone my age probably wouldn't want the information to be incredibly detailed and long but more key phrased and engaging. (\#18) Not being treated like a child when I'm not is a pilg thing though. If they don't respect me what's the point? The service did not understand and I ended up giving up seeking help. (\#4) 
wait times ranged from a few days to a few months

\section{Location}

- Cф-location with addiction services or problem gambling. Could be co-located with a university counselling service

- Access to advice and screening from primary care providers. Facilitate opportunistic interventions.

- Ayailable weekends and in the evenings.

- If online there was a preference that the person had some local context.

\section{Modality}

- Multiple modalities including faceto-face and online. Need for

flexible, convenient and discrete access as required. Online or face to face individual or group treatment.

- Smart phone access for tracking błhaviour and getting help as need and a website for screening and information.

Ability to Direct fees

pay for suppord Exper Expectation that there would be
cost to attend a treatment. The recommend tiered approach based on income. For students the reasonable amount per session was around $\$ 20$ and for those

- I qm a student and currently not working, so I

- If it was closer to exam time I'd be a bit more desperate and therefore want help as quickly as
p ssible, but other times through more relaxed
pqrts of the year I'd be willing to wait a week or
two. (\#7) d sperate and therefore want help as quickly as
p ssible, but other times through more relaxed
p $\mathrm{rts}$ of the year I'd be willing to wait a week or
two. (\#7) d sperate and therefore want help as quickly as
pssible, but other times through more relaxed
pфrts of the year I'd be willing to wait a week or
two. (\#7) desperate and therefore want help as quickly as
p ssible, but other times through more relaxed
pqrts of the year I'd be willing to wait a week or
two. (\#7)

b申cause I'm more used to typing online, it allows me to think through my words as I'm speaking for clear communication and reading replies is easier than listening to them. Online communication is great when you can't make it into town easily. (\#2)

I think maybe an online session chat is the best. Most of people who are addicted to video game are shy, introvert etc. it is hard for them to actually come out and seek help. (\#16)

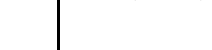


engage C Chfidential, private and capacityS $\phi m e$ pre-involvement lead-up (such as with for anonymity. Culturally support appropriate questions like the ones we talked about earlier)

- Pre-treatment screening so as to straight into tackling the problem from the first nфt waste time

tine I met them. (\#3)

- Multiple modalities (face to face, For me I feel like confidentiality is the most group, online) and blended treatment (different types of service options such as peer and one-to-one). Single session or ongoing treatment.

- Peer support forums moderated by a professional

- Aple to be tailored to need inpportant which getting help with mental disorder. I want to keep it as secret as possible and sometime you don't even want the professional to know you cause sometime you feel insecure about yourself. (\#16)

Definitely confidentiality, nobody likes the idea of others thinking they have a problem. But by this I don't just mean private counseling but (varying intensity from screening a ttually setting up appointments and things like and drop in to residential care) that. (\#7)

- Resources include online and - I think building the skills and confidence myself, w fitten materials.

\section{Treatment needs}

- Skills development for relationships and social with the help of your tools, will help me go further with gaming reduction. (\#10)

- A countability and some form of consultation wpuld be good. Booklets or handouts containing tips would be good, maybe sifuations, emotional intelligence, sdmething regarding logging time online, or time and stress management and time spent doing other stuff, and some form of sleep hygiene. online based community with others. (\#2)

- Tyeat underlying comorbidities - I'ye been trying to tackle other elements around such as impulsivity, substance usea diction such as alcohol and marijuana but the (alcohol, cannabis), depression truth is I have an addictive personality that and anxiety.

extends to many areas of my life. (\#4)

- Enhance self-efficacy and belief-inSфmeone who understands game culture. I feel capacity to change. Support increased accountability, commitment, goal setting, urge couldn't relate to the person on this topic. (\#7) management and self-monitoring.

\section{Quality of providers}

- Professional but not formal with demonstrated unconditional pфsitive regard. Facilitate erppathic and approachable partnerships.

- The provider should understand gaming culture and technology and be an expert in treatment of gaming

\section{Adequacy of service}

- Participants should be satisfied w.th the quality of service and experience the treatment as effective.

The ability to seek support was informed predominantly by beliefs that treatment-seeking for GD was inappropriate, low knowledge of service options and that services would not be willing or able to respond 
(33\% of statements). Participants perceived that the individual should be able to solve the problem themselves and that the issue was not as serious as other conditions. Informing this view was social group responsivity that either minimised the problem or suggested the solution was simple ('just stop'). Participants reported feeling embarrassed at having to seek help and to have to tell their friends that they needed to cut back on gaming for a while. There was also a perception that health resources would be made available according to the burden of disease where gaming was less of a priority concern (due to it not being associated with serious harm). Participants indicated limited knowledge of treatment options and their benefits. Some participants who had previous experience with addiction or health care services reported concerns on the appropriateness of service models that were either adolescent-centric (thereby being more family-centred in their approach) or focused on a disease model of addiction (abstinence over harm minimisation).

The ability to reach services referred to the ease of access and location where they are most needed (22\% of statements). The absence of a service system for GD was highlighted in this theme whereby participants who had sought treatment from addiction or private providers either had to travel long distances to seek help or instead sought help online from international sources. They expressed that help should be in a convenient physical location such as co-located with university or other health care services, including those that address comorbidities such as alcohol and cannabis use. There was a preference for a range of different modalities for treatment spanning online and face-to-face as well as individual and group options. Online treatment was especially attractive for its convenience, less time to access or engage with, allowed participants to type instead or talk (which was preferable initially for introverted help-seekers). There was also a view that some service options should be local, immediate, and available 24/7, which would help to address urgent issues before they became desperate, such as the need to decrease gaming time during exams. Some participants referred to the need for treatment providers to understand the wider context of gaming culture.

The ability to pay related to the direct costs of treatment as well as other facilitating factors $(10 \%$ of statements). There was a preference for direct costs to be assessed according to employment status and income. For those with low income there was an expectation of paying around NZ\$20p/h for the service and upwards of NZ\$100p/h for those that were employed. Factors impacting on ability to pay included recent transitions from home to independent living arrangements. Other factors included over-spending on gaming such as the frequent purchase of loot boxes, new games and other products such as cards and tokens.

The ability to engage with a service related to the quality of fit between client need and the content, quality and delivery modality of treatment ( $26 \%$ of statements). There was a view that services should be tailored according to the amount of treatment needed and the presenting problem. The range of treatment needs was extensive and included gaming focused needs such as enhancing self-efficacy, competency and skill development in limiting or reducing gaming. Treatment need also included comorbidities such as addiction (cannabis and alcohol) as well as depression and anxiety. Other areas requiring assistance included sleep and stress management, social skills, and time management. The 
perceived quality of the treatment and relationship with the provider was perceived as important and this focused on the development of rapport through unconditional positive regard with a general empathic and non-judgemental approach.

\section{Service system components}

Figure 2 depicts the five components of an accessible health care system response. The approachability of services related to the importance of information on GD, screening tools and the provision of service information. There was a need for evidence-based information on the nature and risk of GD. This information would be ideally supported by valid and reliable screening tools that can be self-administered or integrated into treatment phases. Participants expressed confusion and uncertainty as to whether they really had a problem, indicating a need for clear feedback on problem severity and prognosis. While there are currently few community-based treatment options, participants indicated a need for clear service information that could guide decisions about service options based on level of severity as well as modality of delivery.

Acceptability of services involved themes of responsivity and specificity, and values of partnership and mutual respect. Participants expressed a need for high quality specialist gaming services that have indepth knowledge of gaming and its prevention and treatment. Responsive services would value treatment-seeking for gaming and promote this as a normal and reasonable response to these issues. Gaming support and services should be established for adolescent and adult gamers and avoid a 'onesize-fits-most/all' approach. Clinical staff should be experienced in working with young people and have relevant gaming knowledge.

The availability of services related to flexible access and location of the provider. Participants expressed a need for a multi-modal system that supported online, smartphone, and face-to-face treatment accessible in self-help, individual and group-based formats. Participants expressed that services should be available after-hours including evenings and weekends as well as located in or near health care centres or universities. Where travel was required, services should be able to be accessed via public transport and ideally located in each major centre. The affordability of services was proposed to be income-based and should represent value for money. Service costs were proposed as ranging from \$20 upwards depending on private or public funding.

Services should be appropriate to meet the needs of a potentially heterogeneous group of gamers and their family. To be appropriate, a patient-centred approach should include valid and reliable screening tools that can inform treatment plans and the selection of modality and intensity of treatment. This means supporting a transition between service types and intensity whereby individuals are able to alter care plans and arrangements as required. Co-locating treatment within mental health services would ensure that co-morbidities were addressed however there should be an option whereby the treatment primarily is focused on gaming specific issues. The workforce should have an in-depth knowledge of gaming and addictions including gaming culture and its comorbidities. There is also a need to consider the role and involvement of lived experience and how those that have recovered can support others. 
Identified clinical approaches included the delivery of urge management, relapse prevention and psychosocial rehabilitation via replacement activities. Services should be routinely assessed for quality and compliance with standards with these evaluations available to all clients.

\section{Discussion}

The aim of this study was to understand the health care needs of individuals with GD. We applied a patient-centred framework for conceptualising the components of a support system for the identification, early intervention and treatment of GD. Gamers' perceptions and experiences reflected the components of the framework in terms of facilitators and barriers to the ability to perceive, seek, reach, pay and engage with treatment. This approach identified critical issues in GD healthcare that also arise across the spectrum of addictive disorders (e.g., barriers to help seeking shame, stigma and health beliefs around the seriousness and susceptibility of having a problem) as well as use of health care more broadly (being able to navigate, locate and reach support and service options). The second aim was to identify the components of a healthcare system informed by the framework while accounting for participant expectations and need. Critical discussion of this system included the need for transdiagnostic treatments that could address a range of comorbidities including mood disorders, substance use, relationship difficulties, social skills and adaptive functioning.

Gaps in health care identified in this study related to the adequacy of screening and assessment, access to evidence-based treatment and routine outcome monitoring. Screening and assessment has been identified as a major problem in GD research that has a direct impact on service ability to provide valid and reliable feedback to gamers $(23,60)$. Inconsistencies in definitions and screening are a barrier to offering services that rely on brief information-gathering to identify client needs, and to developing consumer trust in the service offered. In terms of access to evidence-based treatment, promising work is being conducted in the area of structured cognitive-behavioural therapy programs (Kim et al., 2012; Wölfling et al., 2019). However, there are currently few RCTs for GD suggesting that the development of an evidence-based treatment model is some way in the future $(36,61)$.

Some participants expressed uncertainty about the legitimacy of GD as a mental health condition. This uncertainty reflected a belief that the problem could be self-managed and was really just a lack of selfcontrol and motivation to change. These concerns are in part consistent with the broader issue of the concept of problem gaming $(2,10-18)$ and highlight the need to develop a diverse range of support options. Participants in the current study were accessing an internet-delivered self-directed programme which was designed to support autonomy and skill building (53). Park et al. reported significant reductions in time spent gaming and improvements to severity of gaming symptoms and general wellbeing. Other studies also indicate gamers engage with a broad range of self-managed cognitive and behavioural strategies to limit their gaming (62) and are able to adhere to personal change goals (63). The current study highlights the need to consider a stepped care service system that is inclusive of lowseverity (e.g., brief interventions such as personalised normative feedback) and low-intensity service 
options (e.g., self-directed treatments) as well as options that are more intensive (e.g., face-to-face services).

A limitation of the field is the absence of quality information on people across the continuum of severity, including case reports and clinical data. Routine outcome monitoring has the potential to address this problem as well as provide insight into the scope of the problem, patterns of use, and responsivity to different treatments (64). Ideally, routine outcome monitoring is informed through international consensus on a minimum dataset with a set of recommendations for clinical outcome and process measures. In particular, there is a need to better understand how problematic gaming may intersect with issues of comorbidity, such as mood disorders, and how such considerations may influence case formulation and treatment planning. Less is known about the efficacy of structured treatments for GD, including psychological and pharmacological treatment, in the context of other mental disorders, because such issues are often excluded from trials and other intervention studies.

This is the first study to consider the optimal components of a service system for GD from a holistic perspective but this work had several limitations. First, the study involved a self-selected sample of helpseeking adults who provided qualitative data. Qualitative studies are not intended to be generalisable and in the current study this data were analysed to identify patterns in help-seeking preferences and experiences. These patterns were mapped onto an established model outlining the potential components of a comprehensive health care system. Future research should identify the relevance, applicability and generalisability of these findings using quantitative methodologies as well as consensus-based approaches to responding to GD. Second, we believe that the model reflects the wide range of issues that need to be considered when establishing a co-ordinated clinical response to GD. However, the nature of the sample means there would be additional nuance needs to be incorporated (e.g., cultural considerations). Finally, participants in this study were motivated to change their gaming behaviour and were in the process of seeking help. Future research should examine the perspective of gamers across the continuum including those that have a problem but do not want to change as well as those who have lived experience of a range of different treatment services.

\section{Conclusions}

A comprehensive health care approach for gaming disorder is currently in its infancy and there are numerous service and support access and delivery issues still to be resolved. A challenge that has received relatively less attention has been the issue of treating GD and its comorbid disorders. Our findings are consistent with lessons from the gambling field that suggest treatment approaches need to to be tailored according readiness to change (65) and specific needs such as co-occuring issues. The pathways model of problem gambling outlines three different profiles of gamblers (i) behaviourally conditioned (ii) emotionally vulnerable (iii) impulsive/anti-social (66). The model recommends treatment is matched accordingly whereby those who are behaviourally conditioned may benefit from less intensive brief interventions. In comparison those who present with comorbidities such as mood disorders would likely benefit from more intensive treatment that covers a range of different presenting issues. The 
proposed model in the current study is consistent with this approach in terms of recommending a range of different service options that are tailored to the needs of individuals. Currently, health care systems for GD lack a stepped care approach whereby a gamer can transition between levels of treatment as needed (23).

The current study has identified issues associated with the establishment of a service system for GD. A key issue with establishing a service system is the identification of an appropriate workforce who has the skills and expertise to administer the range of different service responses. Future research might identify existing capacity and how it aligns with the expertise identified in the current study.

\section{List Of Abbreviations}

GD: Gaming Disorder

DSM-5: Diagnositic and Statistical Manual Version 5

ICD-11: International Classification of Diseases

CBT: Cognitive and Behavioural Therapy

GAS: Gaming Addiction Scale

\section{Declarations}

Ethics approval and consent to participate: All procedures performed in studies involving human participants were in accordance with the ethical standards of the institutional and/or national research committee and with the 1964 Helsinki declaration and its later amendments or comparable ethical standards. The research was approved by the University of Auckland Human Participants Ethics Committee (022614). Online informed consent was obtained from all participants after reviewing the study purpose and aims.

Consent for publication: Not applicable

Competing interests: The authors have no competing interests to declare in relation to this article.

Availability of data and materials: The datasets and materials used and analysed during the current study are available from the corresponding author on reasonable request.

Funding: This research was undertaken as part of a Health Research Council early career grant awarded to the senior author (17/548). Additional funding for participant recruitment and remuneration was provided by Internet NZ. The funders had no role in the design or conduct of the study or decision to publish the findings. 
Authors' contributions: Author SR designed the study and sought the funding. Author JP conducted literature searches and prepared the first draft of the manuscript. Author JP and SR conducted the analysis. All authors contributed to and have approved the final manuscript.

Acknowledgements: We would like to thank the Change Strategies Project team for their assistance with the recruitment of participants.

\section{References}

1. Ferguson CJ, Coulson M, Barnett J. A meta-analysis of pathological gaming prevalence and comorbidity with mental health, academic and social problems. Journal of psychiatric research. 2011;45(12):1573-8.

2. Przybylski AK, Weinstein N, Murayama K. Internet gaming disorder: Investigating the clinical relevance of a new phenomenon. American Journal of Psychiatry. 2017;174(3):230-6.

3. Mihara S, Higuchi S. Cross-sectional and longitudinal epidemiological studies of I nternet gaming disorder: A systematic review of the literature. Psychiatry and clinical neurosciences. 2017;71(7):42544.

4. Stevens MW, Dorstyn D, Delfabbro PH, King DL. Global prevalence of gaming disorder: A systematic review and meta-analysis. Australian \& New Zealand Journal of Psychiatry. 2020:0004867420962851.

5. Saunders JB, Hao W, Long J, King DL, Mann K, Fauth-Bühler M, et al. Gaming disorder: Its delineation as an important condition for diagnosis, management, and prevention. Journal of behavioral addictions. 2017;6(3):271-9.

6. Petry NM, Rehbein F, Gentile DA, Lemmens JS, Rumpf HJ, Mößle T, et al. An international consensus for assessing internet gaming disorder using the new DSM-5 approach. Addiction. 2014;109(9):1399406.

7. Long J, Liu T, Liu Y, Hao W, Maurage P, Billieux J. Prevalence and correlates of problematic online gaming: a systematic review of the evidence published in Chinese. Current Addiction Reports. 2018;5(3):359-71.

8. Andreassen CS, Billieux J, Griffiths MD, Kuss DJ, Demetrovics Z, Mazzoni E, et al. The relationship between addictive use of social media and video games and symptoms of psychiatric disorders: A large-scale cross-sectional study. Psychology of Addictive Behaviors. 2016;30(2):252.

9. Wang HR, Cho H, Kim D-J. Prevalence and correlates of comorbid depression in a nonclinical online sample with DSM-5 internet gaming disorder. Journal of affective disorders. 2018;226:1-5.

10. Aarseth E, Bean AM, Boonen H, Colder Carras M, Coulson M, Das D, et al. Scholars' open debate paper on the World Health Organization ICD-11 Gaming Disorder proposal. Journal of Behavioral Addictions. 2017;6(3):267-70.

11. Billieux J, Flayelle M, Rumpf H-J, Stein DJ. High involvement versus pathological involvement in video games: A crucial distinction for ensuring the validity and utility of gaming disorder. Current 
Addiction Reports. 2019;6(3):323-30.

12. Dullur P, Starcevic V. Internet gaming disorder does not qualify as a mental disorder. Australian and New Zealand Journal of Psychiatry. 2018;52(2):110-1.

13. Higuchi S, Nakayama H, Mihara S, Maezono M, Kitayuguchi T, Hashimoto T. Inclusion of gaming disorder criteria in ICD-11: A clinical perspective in favor: Commentary on: Scholars' open debate paper on the World Health Organization ICD-11 Gaming Disorder proposal (Aarseth et al.). Journal of Behavioral Addictions. 2017;6(3):293-5.

14. Kardefelt-Winther D. A critical account of DSM-5 criteria for internet gaming disorder. Addiction Research \& Theory. 2015;23(2):93-8.

15. Kardefelt-Winther D. Conceptualizing I nternet use disorders: A ddiction or coping process? Psychiatry and Clinical Neurosciences. 2017;71(7):459-66.

16. King DL, Delfabbro PH, Potenza MN, Demetrovics Z, Billieux J, Brand M. Internet gaming disorder should qualify as a mental disorder. Australian \& New Zealand Journal of Psychiatry. 2018;52(7):615-7.

17. Rumpf H-J, Achab S, Billieux J, Bowden-Jones H, Carragher N, Demetrovics Z, et al. Including gaming disorder in the ICD-11: The need to do so from a clinical and public health perspective: Commentary on: A weak scientific basis for gaming disorder: Let us err on the side of caution (van Rooij et al., 2018). Journal of behavioral addictions. 2018;7(3):556-61.

18. van RooijAntonius J, FergusonChristopher J, CarrasMichelle C, BeanAnthony M, Helmersson B, EtchellsPeter J, et al. A weak scientific basis for gaming disorder: Let us err on the side of caution. Journal of behavioral addictions. 2018.

19. World Health Organisation. 6C51 Gaming Disorder. Retrieved online: https://icd.who.int/browse11/Im/en\#/http://id.who.int/icd/entity/1448597234.2019.

20. King DL, Delfabbro PH. Prevention and policy related to Internet gaming disorder. Current Addiction Reports. 2017;4(3):284-92.

21. Vondráčková P, Gabrhelik R. Prevention of Internet addiction: A systematic review. Journal of behavioral addictions. 2016;5(4):568-79.

22. Xiang Y-T, Jin Y, Zhang L, Li L, Ungvari GS, Ng CH, et al. An Overview of the Expert Consensus on the Prevention and Treatment of Gaming Disorder in China (2019 Edition). Neuroscience Bulletin. 2020:1-4.

23. Rumpf H-J, Bischof A, Bischof G, Besser B, Brand D, Rehbein F. Early Intervention in Gaming Disorder: What Can We Learn from Findings in the Substance Abuse Field? Current Addiction Reports. 2018;5(4):511-6.

24. King DL, Delfabbro PH, Wu AM, Doh YY, Kuss DJ, Pallesen S, et al. Treatment of Internet gaming disorder: An international systematic review and CONSORT evaluation. Clinical Psychology Review. 2017;54:123-33.

25. Zajac K, Ginley MK, Chang R. Treatments of internet gaming disorder: a systematic review of the evidence. Expert review of neurotherapeutics. 2020;20(1):85-93. 
26. Zajac K, Ginley MK, Chang R, Petry NM. Treatments for Internet gaming disorder and Internet addiction: A systematic review. Psychology of Addictive Behaviors. 2017;31(8):979-94.

27. Kuss DJ, Lopez-Fernandez O. Internet addiction and problematic Internet use: A systematic review of clinical research. World journal of psychiatry. 2016;6(1):143.

28. Shek DT, Yu L. Longitudinal impact of the project PATHS on adolescent risk behavior: what happened after five years? The Scientific World Journal. 2012;2012.

29. Yeun YR, Han SJ. Effects of psychosocial interventions for school-aged children's internet addiction, self-control and self-esteem: meta-analysis. Healthcare Informatics Research. 2016;22(3):217-30.

30. Wang Q, Ren H, Long J, Liu Y, Liu T. Research progress and debates on gaming disorder. General psychiatry. 2019;32(3).

31. King DL, Delfabbro PH, Doh YY, Wu AM, Kuss DJ, Pallesen S, et al. Policy and prevention approaches for disordered and hazardous gaming and Internet use: An international perspective. Prevention Science. 2018;19(2):233-49.

32. Davies B, Blake E. Evaluating existing strategies to limit video game playing time. IEEE computer graphics and applications. 2016;36(2):47-57.

33. Király O, Griffiths MD, King DL, Lee H-K, Lee S-Y, Banyai F, et al. Policy responses to problematic video game use: A systematic review of current measures and future possibilities. Journal of Behavioral Addictions. 2018;7(3):503-17.

34. Stevens MW, King DL, Dorstyn D, Delfabbro PH. Cognitive-behavioral therapy for Internet gaming disorder: A systematic review and meta-analysis. Clinical psychology \& psychotherapy. 2019;26(2):191-203.

35. Costa S, Kuss D. Clinical Practices for Gaming Disorders: A Systematic Review. Frontiers in psychology. 2019;10:578.

36. Wölfling K, Müller KW, Dreier M, Ruckes C, Deuster O, Batra A, et al. Efficacy of short-term treatment of internet and computer game addiction: a randomized clinical trial. JAMA psychiatry. 2019;76(10):1018-25.

37. King DL, Billieux J, Carragher N, Delfabbro PH. Face validity evaluation of screening tools for gaming disorder: Scope, language, and overpathologizing issues. Journal of behavioral addictions. 2020;9(1):1-13.

38. King DL, Chamberlain SR, Carragher N, Billieux J, Stein D, Mueller K, et al. Screening and assessment tools for gaming disorder: A comprehensive systematic review. Clinical Psychology Review. 2020;77:101831.

39. Muller KW, Beutel ME, Wolfling K. Decreased Occupational Functioning and Increased Physical Health Complaints in Treatment Seekers with Internet-Related Disorders: Compared to Patients with Gambling Disorder. European addiction research. 2019;25(5):229-37.

40. Feng W, Ramo D, Chan S, Bourgeois J. Internet gaming disorder: trends in prevalence 1998-2016. Addictive behaviors. 2017;75:17. 
41. Thege BK, Woodin EM, Hodgins DC, Williams RJ. Natural course of behavioral addictions: A 5-year longitudinal study. BMC psychiatry. 2015;15(1):4.

42. Grant BF, Goldstein RB, Saha TD, Chou SP, Jung J, Zhang H, et al. Epidemiology of DSM-5 alcohol use disorder: results from the National Epidemiologic Survey on Alcohol and Related Conditions III. JAMA psychiatry. 2015;72(8):757-66.

43. Yeh Y-C, Wang P-W, Huang M-F, Lin P-C, Chen C-S, Ko C-H. The procrastination of Internet gaming disorder in young adults: The clinical severity. Psychiatry research. 2017;254:258-62.

44. Müller KW, Beutel M, Egloff B, Wölfling K. Investigating risk factors for Internet gaming disorder: a comparison of patients with addictive gaming, pathological gamblers and healthy controls regarding the big five personality traits. European addiction research. 2014;20(3):129-36.

45. Lee YS, Son JH, Park JH, Kim SM, Kee BS, Han DH. The comparison of temperament and character between patients with internet gaming disorder and those with alcohol dependence. Journal of Mental Health. 2017;26(3):242-7.

46. Burleigh TL, Griffiths MD, Sumich A, Stavropoulos V, Kuss DJ. A systematic review of the cooccurrence of Gaming Disorder and other potentially addictive behaviors. Current Addiction Reports. 2019;6(4):383-401.

47. King DL, Adair C, Saunders JB, Delfabbro PH. Clinical predictors of gaming abstinence in helpseeking adult problematic gamers. Psychiatry research. 2018;261:581-8.

48. Lau C, Stewart SL, Sarmiento C, Saklofske DH, Tremblay PF. Who is at risk for problematic video gaming? Risk factors in problematic video gaming in clinically referred Canadian children and adolescents. Multimodal Technologies and Interaction. 2018;2(2):19.

49. Humphreys G. Sharpening the focus on gaming disorder. World Health Organization Bulletin of the World Health Organization. 2019;97(6):382-3.

50. Drummond A, Sauer JD, Ferguson CJ, Hall LC. The relationship between problem gambling, excessive gaming, psychological distress and spending on loot boxes in Aotearoa New Zealand, Australia, and the United States-A cross-national survey. Plos one. 2020;15(3):e0230378.

51. Keough B. Video game addicts seek help from gambling foundation as most health board don't provide treatment. 2018 [Available from: https://www.stuff.co.nz/national/health/109179959/videogame-addicts-seek-help-from-gambling-foundation-as-most-health-boards-dont-provide-treatment.

52. Dullur P, Hay P. Problem Internet use and Internet gaming disorder: A survey of health literacy among psychiatrists from Australia and New Zealand. Australasian Psychiatry. 2017;25(2):140-5.

53. Park JJ, Booth N, Bagot KL, Rodda SN. A brief internet-delivered intervention for the reduction of gaming-related harm: A feasibility study. Computers in Human Behavior Reports. 2020;2:100027.

54. Lemmens JS, Valkenburg PM, Peter J. Development and validation of a game addiction scale for adolescents. Media psychology. 2009;12(1):77-95.

55. Weinstock J, Whelan JP, Meyers AW. Behavioral assessment of gambling: an application of the timeline followback method. Psychological Assessment. 2004;16(1):72-80. 
56. Sobell LC, Sobell MB. Timeline Follow-Back. In: Litten R, Allen J, editors. Measuring Alcohol Consumption: Humana Press; 1992. p. 41-72.

57. Kessler RC, Andrews G, Colpe LJ, Hiripi E, Mroczek DK, Normand S-L, et al. Short screening scales to monitor population prevalences and trends in non-specific psychological distress. Psychological medicine. 2002;32(6):959-76.

58. Levesque J-F, Harris MF, Russell G. Patient-centred access to health care: conceptualising access at the interface of health systems and populations. International journal for equity in health. 2013;12(1):18.

59. Hsieh H-F, Shannon SE. Three approaches to qualitative content analysis. Qualitative health research. 2005;15(9):1277-88.

60. King DL, Haagsma MC, Delfabbro PH, Gradisar M, Griffiths MD. Toward a consensus definition of pathological video-gaming: A systematic review of psychometric assessment tools. Clinical psychology review. 2013;33(3):331-42.

61. Kim SM, Han DH, Lee YS, Renshaw PF. Combined cognitive behavioral therapy and bupropion for the treatment of problematic on-line game play in adolescents with major depressive disorder. Computers in human behavior. 2012;28(5):1954-9.

62. Rodda SN, Booth N, Vacaru M, Knaebe B, Hodgins DC. Behaviour change strategies for internet, pornography and gaming addiction: A taxonomy and content analysis of professional and consumer websites. Computers in Human Behavior. 2018;84:467-76.

63. King DL, Kaptsis D, Delfabbro PH, Gradisar M. Craving for Internet games? Withdrawal symptoms from an 84-h abstinence from massively multiplayer online gaming. Computers in Human Behavior. 2016;62:488-94.

64. King DL, Delfabbro PH. Internet gaming disorder treatment: a review of definitions of diagnosis and treatment outcome. Journal of clinical psychology. 2014;70(10):942-55.

65. Rodda SN, Lubman DI, lyer R, Gao CX, Dowling NA. Subtyping based on readiness and confidence: the identification of help-seeking profiles for gamblers accessing web-based counselling. Addiction (Abingdon, England). 2015;110(3):494-501.

66. Blaszczynski A, Nower L. A pathways model of problem and pathological gambling. Addiction. 2002;97(5):487-99.

\section{Figures}




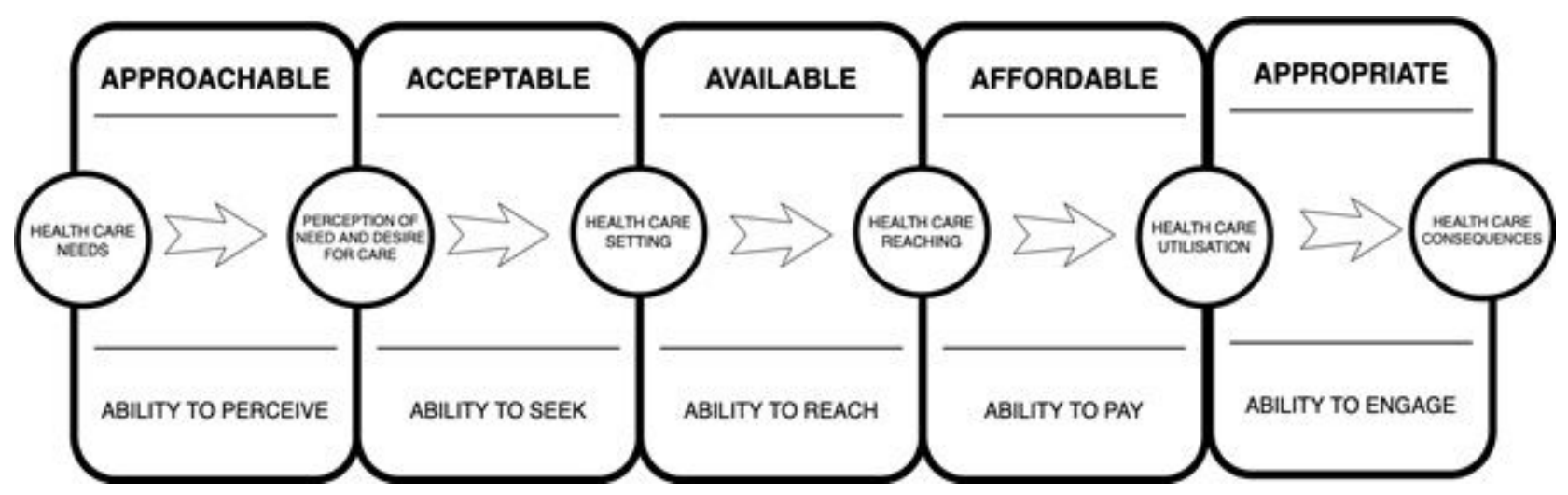

Figure 1

\section{Adapted conceptual framework of access to health care}

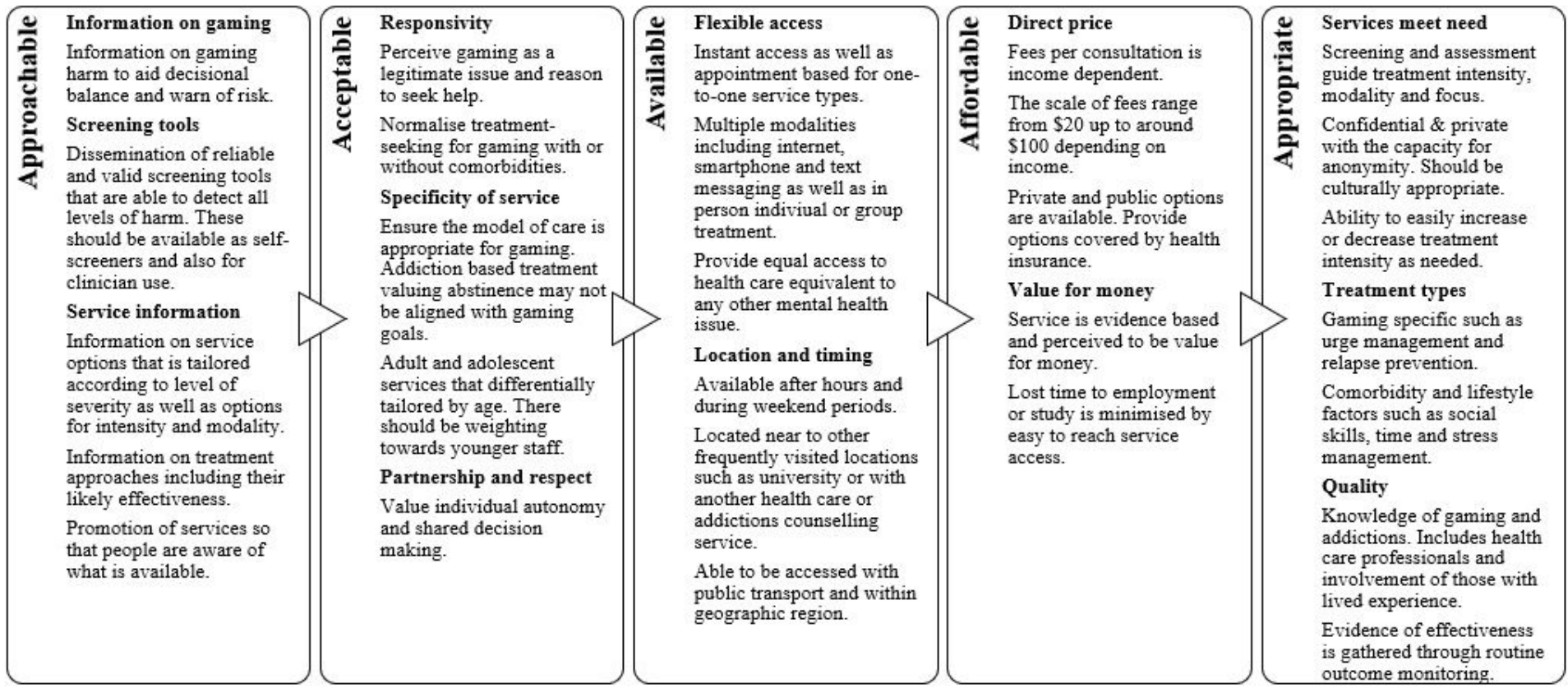

Figure 2

Key components of a patient-centred approach to prevention, early intervention, and treatment of GD 